\title{
Planar open books, monodromy factorizations and symplectic fillings
}

\author{
Olga Plamenevskaya \\ JEREMY VAN HORN-MORRIS
}

\begin{abstract}
We study fillings of contact structures supported by planar open books by analyzing positive factorizations of their monodromy. Our method is based on Wendl's theorem on symplectic fillings of planar open books. We prove that every virtually overtwisted contact structure on $L(p, 1)$ has a unique filling, and describe fillable and nonfillable tight contact structures on certain Seifert fibered spaces.
\end{abstract}

57R17; 53D35

\section{Introduction}

By Giroux's theorem [9], a contact 3-manifold $(Y, \xi)$ is Stein fillable if and only if it is compatible with an open book $(S, \phi)$ whose monodromy $\phi$ can be represented as a product of positive Dehn twists. Given a factorization of the monodromy into a product $\phi=D_{\alpha_{1}} \cdots D_{\alpha_{k}}$ of positive Dehn twists around homologically nontrivial curves $\alpha_{1}, \ldots, \alpha_{k}$, we can construct a Stein filling as an allowable Lefschetz fibration over $D^{2}$ with fiber $S$, with vanishing cycles corresponding to $\alpha_{1}, \ldots, \alpha_{k}$. (We say that a Lefschetz fibration is allowable if all vanishing cycles are homologically nontrivial in their fibers.) Conversely, if $X$ is a Stein manifold whose boundary is $(Y, \xi)$, then $X$ has a structure of an allowable Lefschetz fibration by Akbulut and Ozbagci [1]. The boundary $\partial X=Y$ has an open book decomposition whose monodromy is a product of positive Dehn twists around curves corresponding to the vanishing cycles; this open book is compatible with the contact structure $\xi$ by Plamenevskaya [25]. Thus, Stein fillings of $(Y, \xi)$ correspond to positive factorizations of monodromies of compatible open books. However, to detect nonfillability or to classify all Stein fillings, one would have to consider all possible open books compatible with $\xi$.

The situation is much simpler for contact structures compatible with planar open books, thanks to the following recent result of Chris Wendl. 
Theorem 1.1 (Wendl [27]) Suppose that $(Y, \xi)$ admits a planar open book decomposition. Then every strong symplectic filling $(X, \omega)$ of $(Y, \xi)$ is symplectic deformation equivalent to a blow-up of an allowable Lefschetz fibration compatible with the given open book for $(Y, \xi)$.

In particular, Wendl's theorem implies that every Stein filling of $(Y, \xi)$ is diffeomorphic (and even symplectic deformation equivalent) to an allowable Lefschetz fibration compatible with the given planar open book; to classify fillings or to prove nonfillability, it suffices to study positive factorizations of the given monodromy. Even so, enumerating positive factorizations for a given element of the mapping class is in general a very hard question. However, we are able to analyze certain simple monodromies by means of elementary calculations in the abelianization of the mapping class group of the planar surface. In this paper, we give two applications: first, we complete the classification of fillings for tight lens spaces $L(p, 1)$, second, we study the fillability question for certain tight Seifert fibered spaces.

Theorem 1.2 Every virtually overtwisted contact structure on $L(p, 1)$ has a unique Stein filling (up to symplectic deformation), which is also its unique weak symplectic filling (up to symplectic deformation and blow-up).

Corollary 1.3 For $p \neq 4$, every tight contact structure on $L(p, 1)$ has a unique Stein filling (up to symplectic deformation), which is also its unique weak symplectic filling (up to symplectic deformation and blow-up).

The above corollary combines Theorem 1.2 together with earlier results, giving a complete description of fillings for tight $L(p, 1)$. (Note that fillings for $L(4,1)$ are also understood due to McDuff [22] and our theorem.) Recall that Eliashberg established uniqueness of a symplectic filling (up to deformation and blow-up) for the standard contact structure on $S^{3}$ [3]. McDuff proved that standard contact structures on $L(p, 1)$ all have unique filling except for $L(4,1)$, which has two fillings, up to blow-up and diffeomorphism [22]. Hind showed that in McDuff's theorem, the Stein filling of $\left(L(p, 1), \xi_{\text {std }}\right)$ is in fact unique up to Stein homotopy [12]. Lisca extended these results to obtain a classification (again up to blow-up and diffeomorphism) of symplectic fillings for arbitrary lens spaces $L(p, q)$ equipped with standard contact structures [18]. (By the standard contact structure on a lens space we mean the quotient of $\left(S^{3}, \xi_{\text {std }}\right)$ by the action of the cyclic group. All of the above results for the standard contact structure obviously extend to its conjugate, thus covering the two universally tight contact structures on $L(p, q)$.) Theorem 1.2 extends classification of fillings in another direction, by a different technique. Our technique also allows to reprove uniqueness of symplectic fillings of $\left(L(p, 1), \xi_{\text {std }}\right)$ for $p \neq 4$. 

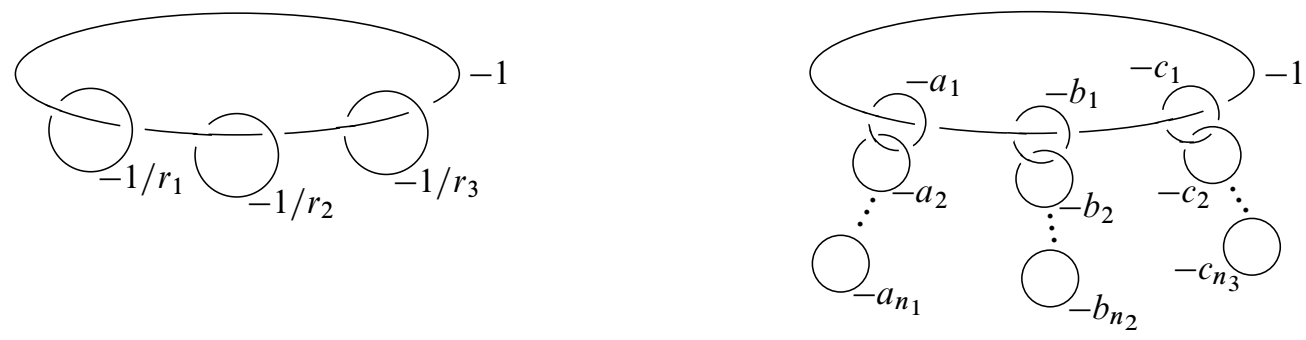

Figure 1: Seifert fibered space $M\left(-1 ; r_{1}, r_{2}, r_{3}\right)$

Our second application concerns fillability of contact structures on Seifert fibered spaces $M\left(-1 ; r_{1}, r_{2}, r_{3}\right)$. (We use this notation for the space given by the surgery diagram of Figure 1; here and throughout the paper, $r_{1}, r_{2}, r_{3}$ are rational numbers between 0 and 1.) Tight contact structures on such manifolds were studied by Ghiggini-LiscaStipsicz [8] and Lisca-Stipsicz [19; 20]; when $r_{1}, r_{2} \geq 1 / 2$, a complete classification of tight contact structures on $M\left(-1 ; r_{1}, r_{2}, r_{3}\right)$ was obtained in [8] (in particular, each of these spaces is known to carry a tight contact structure). Tightness of some of these contact structures was established by means of the Heegaard Floer theory; it was shown in [8] that one of the tight structures on $M(-1 ; 1 / 2,1 / 2,1 / p)$ is nonfillable. (Recall that, in contrast, all tight contact structures on $M\left(0 ; r_{1}, r_{2}, r_{3}\right)$ are fillable; see Ghiggini-Lisca-Stipsicz [7], cf Wu [28]).

It is interesting to determine which of the manifolds $M\left(-1 ; r_{1}, r_{2}, r_{3}\right)$ carry tight, nonfillable contact structures. Wendl's theorem provides a good tool for this investigation, because all tight contact structures on $M\left(-1 ; r_{1}, r_{2}, r_{3}\right)$ admit planar open books, at least in the case $r_{1}, r_{2} \geq 1 / 2$. It turns out that fillability depends, in a rather subtle way, on the arithmetics of the continued fraction expansions of $r_{1}, r_{2}, r_{3}$. Let

$$
-\frac{1}{r_{1}}=\left[a_{1}, a_{2}, \ldots, a_{n_{1}}\right], \quad-\frac{1}{r_{2}}=\left[b_{1}, b_{2}, \ldots, b_{n_{2}}\right], \quad-\frac{1}{r_{3}}=\left[c_{1}, c_{2}, \ldots, c_{n_{3}}\right],
$$

where we adopt the notation

$$
\left[x_{1}, x_{2}, \ldots, x_{n}\right]=-x_{1}-\frac{1}{-x_{2}-\frac{1}{\ddots \cdot-\frac{1}{-x_{n}}}}, \quad x_{i} \in \mathbb{Z}, x_{i} \geq 2 .
$$

Theorem 1.4 Suppose that $r_{1}, r_{2} \geq 1 / 2$. Let $k_{1}, k_{2}$ be such that $a_{1}=a_{2}=\cdots=$ $a_{k_{1}}=2, b_{1}=b_{2}=\cdots=b_{k_{2}}=2$, and $a_{k_{1}+1} \geq 3, b_{k_{2}+1} \geq 3$ (if $k_{1}<n_{1}$, resp. $k_{2}<$ $\left.n_{2}\right)$. Then the space $M\left(-1 ; r_{1}, r_{2}, r_{3}\right)$ carries tight, symplectically nonfillable contact structures if $c_{1}-1>\max \left(k_{1}, k_{2}\right)$; otherwise all tight structures on $M\left(-1 ; r_{1}, r_{2}, r_{3}\right)$ are Stein fillable. 
Our proofs of Theorem 1.2 and Theorem 1.4 use abelian information about the planar mapping class group as provided by a nice group presentation given by Margalit and McCammond [21]. From their presentation we extract invariants from the abelianization which obstruct positive factorizations and hence Stein fillings. These invariants can also determine the possible factorizations in the abelianization, which can be lifted to factorizations in the mapping class group but only up to conjugacy. Outside of some special cases, this isn't enough to pin down the actual factorizations, though perhaps more sensitive representations or a more complete understanding of the planar mapping class group might be able to eliminate these difficulties. To get new results with this method, we need additional tricks: the proof of Theorem 1.2 combines our invariants with some deep topological results.

It is instructive to compare our method in Theorem 1.4 to other proofs of nonfillability. Previous techniques (see eg Lisca [17] and Ghiggini-Lisca-Stipsicz [8]) are all based on some version of gauge theory and the Donaldson theorem, as follows. First one shows, using Seiberg-Witten or Heegaard Floer theory, that all symplectic fillings of a given contact structure must be negative-definite; then the filling is completed to a closed negative-definite 4-manifold, so that by the Donaldson theorem the intersection form of the filling embeds into a standard diagonalizable form over the integers. Finally, existence of such an embedding must be ruled out; often, this is possible because the first Chern class of a Stein filling can be understood in terms of the contact structure. We note that this method is in principle applicable to planar open books, because by Etnyre [5] and Oszváth-Stipsicz-Szabó [24], every symplectic filling of a contact structure $\xi$ supported by a planar open book must be negative-definite, and have the intersection form that embeds into a standard diagonalizable form over the integers; moreover, if $c_{1}(\xi)=0$, then $c_{1}$ of every Stein filling of $\xi$ must be 0 . However, the analysis of possible embeddings of the intersection form can typically be done only in a very limited number of cases. Our analysis of the monodromy factorizations, at least on the level of the abelianization, appears be a lot easier to do, and works in many situations.

Acknowledgements We are grateful to John Etnyre for some helpful conversations, to Dan Margalit and Chris Wendl for helpful correspondence, and to Paolo Lisca and András Stipsicz for their comments on a preliminary version of this paper.

The first author is partially supported by NSF grant DMS-0805836.

\section{Lens spaces $L(p, 1)$ and their fillings}

By Honda's classification of tight contact structures on lens spaces [13], all tight contact structures on $L(p, 1)$ arise as surgeries on Legendrian unknot with $\mathrm{tb}=-p+1$, ie 
the standard Legendrian unknot stabilized $p-2$ times. More precisely, there are $p-1$ tight contact structures $\xi_{1}, \xi_{2}, \ldots \xi_{p-1}$ on $L(p, 1)$, distinct up to isotopy; the contact structure $\xi_{k}$ is the result of Legendrian surgery on the stabilized unknot with $k$ cusps on the left and $p-k$ cusps on the right. Legendrian surgery yields a Stein filling for $\xi_{k}$ which is diffeomorphic to the union of $D^{4}$ and the 2-handle corresponding to the surgery.

Our job is to prove that every Stein filling for a virtually overtwisted $(L(p, 1), \xi)$ is diffeomorphic to the one described above, and that the Stein structure is unique, at least up to symplectic deformation. To do this, we examine factorizations of monodromies of planar open books as products of positive Dehn twists. The question of determining such factorizations in a general mapping class group is very hard (for example, it includes the enumeration of all Lefschetz pencils, half of the information needed to classify minimal symplectic manifolds). While this question in the planar mapping class group looks like it may be simpler, we were unable to solve this problem by looking directly at the planar mapping class group. To make our task easier, we look instead to the abelianization of the mapping class group and solve the analogous problem there; we find all ways to write the image of our mapping class as a sum of images of Dehn twists. This only solves our problem up to conjugation of each factor. While this does solve the problem in some very special cases, it is not enough for our virtually overtwisted lens spaces. To finish the proof here, we need to invoke some powerful theorems in low-dimensional topology. We first show that all the possible open book decompositions with positive factorizations and which represent the same class in the abelianization are obtained from the tight contact $S^{3}$ by Legendrian surgery on a knot with negative Thurston-Bennequin number. We can then apply the surgery characterization of Kronheimer, Mrowka, Ozsváth and Szabó [16] and Legendrian classification of Eliashberg and Fraser [4] of the unknot to achieve our desired result. This reliance on the complete understanding of the unknot means the specific methods used here will not translate easily to other questions about uniqueness of Stein fillings. This should be seen as an indictment of our understanding of the planar mapping class group rather than of the method itself. We hope that a better understanding of the planar mapping class group can push these ideas to further results.

To begin, observe that the virtually overtwisted contact structures are those labeled by $\xi_{2}, \ldots, \xi_{p-2}$ in the above notation. The corresponding Legendrian unknots have stabilizations on both sides. Indeed, a lift of the curve $C$ (on the left of Figure 3) bounds an overtwisted disk in appropriate cover [11, Proposition 11.2.12]. The two universally tight contact structures $\xi_{1}$ and $\xi_{p-1}$ are given by surgeries on the unknot that has all of the stabilizations on the right or on the left. We construct planar open books for $\left(L(p, 1), \xi_{k}\right)$ as follows. The standard Legendrian unknot with tb $=-1$ 
can be represented as the core circle of a page of the open book decomposition of $\left(S^{3}, \xi_{\text {std }}\right)$ with annular pages; to place the stabilized unknot on a page of an open book for $\left(S^{3}, \xi_{\text {std }}\right)$, we stabilize the open book and modify the knot as described in Etnyre [6]; see Figure 2.
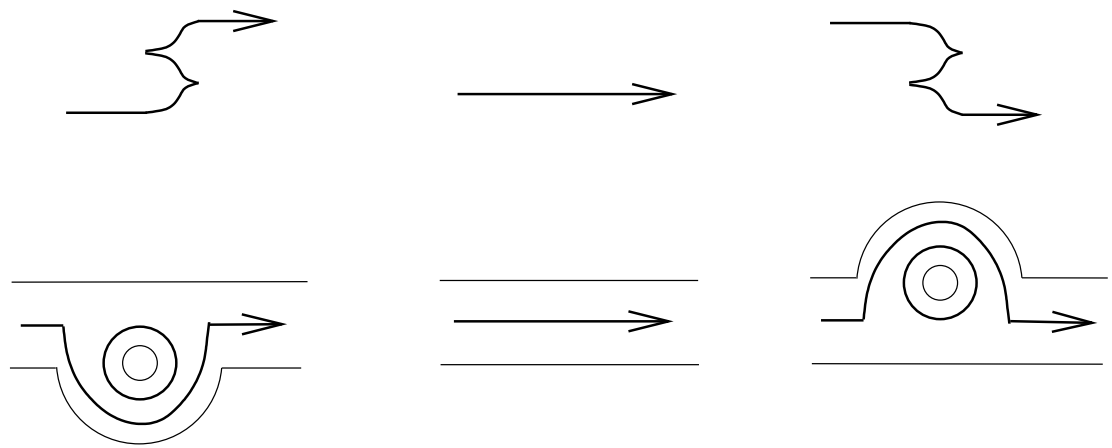

Figure 2: Placing a stabilized knot on a page of an open book

In this setup, the page framing matches the Thurston-Bennequin framing of the Legendrian unknot; performing a positive Dehn twist on the curve representing the unknot, we obtain an open book decomposition corresponding to the result of Legendrian surgery. The resulting open book for $\left(L(p, 1), \xi_{k}\right)$ is shown in Figure 3: the page of the open book is a disk with $n=p-1$ holes, and the monodromy

$$
\Phi=D_{\alpha} D_{\delta_{1}} \cdots D_{\delta_{k-1}} D_{\delta_{k+1}} D_{\delta_{n}} D_{\beta}
$$

is the product of positive Dehn twists $D_{\delta_{i}}$ around each of the holes except the $k$-th, and the positive Dehn twists around the curves $\alpha$ and $\beta$. Here the order of Dehn twists is unimportant, because for a general open book it only matters up to cyclic permutation, and here the boundary twists commute with all other Dehn twists. However, it is convenient to fix notation now:

Convention 2.1 Throughout the paper, we adhere to braid notation for products of Dehn twists: in the expression $D_{\alpha} D_{\beta}, D_{\alpha}$ is performed first.

We first show that any positive factorization of $\Phi$ consists of Dehn twists enclosing the same holes. (In a disk with holes, every simple closed curve separates, and we say that a collection of holes is enclosed by a curve if the holes lie in the component not containing the outer boundary of the disk. Abusing the language, we will often talk about holes enclosed by Dehn twists.) 

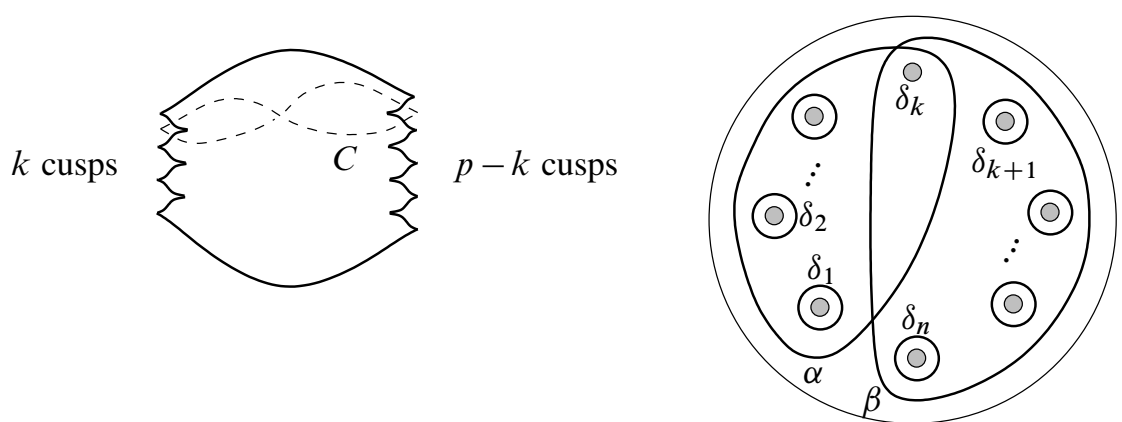

Figure 3: A surgery diagram for the virtually overtwisted contact structure $\xi_{k}$ on $L(p, 1)$. A lift of the curve $C$ (dashed in the picture) bounds an embedded overtwisted disk in a cover of $\left(L(p, 1), \xi_{k}\right)$. On the left, a compatible open book is shown. (Here $p=n+1$.) The monodromy is the product $\Phi=$ $D_{\alpha} D_{\delta_{1}} \cdots D_{\delta_{k-1}} D_{\delta_{k+1}} D_{\delta_{n}} D_{\beta}$ of positive Dehn twists around the pictured curves.

Lemma 2.2 For the open book shown in Figure 3, any positive factorization of the monodromy $\Phi$ must be given by the product of the Dehn twists $D_{\delta_{1}}, \ldots, D_{\delta_{k-1}}$, $D_{\delta_{k+1}}, \ldots D_{\delta_{n}}$, and the Dehn twists $D_{\alpha^{\prime}}$ and $D_{\beta^{\prime}}$ around some curves $\alpha^{\prime}$ and $\beta^{\prime}$, such that $\alpha^{\prime}$ encloses the same holes as $\alpha$, and $\beta^{\prime}$ the same holes as $\beta$.

To prove the lemma, we will need to look closely at the mapping class group of a planar surface; it will be convenient to work with its presentation given by Margalit and McCammond in [21]. (Note: The conventions in [21] are opposite ours and those used by most 4-manifold or symplectic topologists; they use left-handed Dehn twists as the positive generators of the presentation.)

Consider a round disk with punctures arranged at the vertices of a regular $n$-gon contained in the disk; let the holes be small neighborhoods of the punctures, and denote by $\mathbf{D}_{n}$ the resulting disk with $n$ holes. We say that a simple closed curve in $\mathbf{D}_{n}$ is convex if it is isotopic to the boundary of the convex hull of some of the holes; a Dehn twist around a convex curve is said to be convex. By [21], the mapping class group has a presentation with generators given by all (distinct) convex Dehn twists, and relations of the following two types. The first type states that Dehn twists around disjoint curves commute. The second type consists of all possible lantern relations; a lantern relation is the relation of the sort $D_{A} D_{B} D_{C} D_{A \cup B \cup C}=D_{A \cup B} D_{B \cup C} D_{A \cup C}$. Here $A, B, C$ are disjoint collections of holes, $D_{A}, D_{A \cup B}$, etc, are convex Dehn twists around the curves enclosing the corresponding sets of holes, and the collections $A, B$, and $C$ are such that the cyclic clockwise ordering of all the holes in $A \cup B \cup C$, induced from their convex position on the disk, is compatible with the ordering where we list all 
holes from $A$ in their cyclic order, then all holes from $B$, then all holes from $C$. Each collection $A, B, C$ may contain one or more holes; the ordering condition ensures that the Dehn twists are performed around curves arranged as in the usual lantern relation. See [21] for details.

Proof of Lemma 2.2 First, observe that although the set of holes enclosed by a simple closed $\gamma$ does not determine $\gamma$ up to isotopy, it determines, up to conjugacy, the class of the Dehn twist $D_{\gamma}$ in Map $\mathbf{D}_{n}$. (If $\gamma, \gamma^{\prime}$ enclose the same holes, the conjugacy between $D_{\gamma}$ and $D_{\gamma^{\prime}}$ is given by the diffeomorphism $h \in \operatorname{Map} \mathbf{D}_{n}$ that takes $\gamma$ to $\gamma^{\prime}$.) Consequently, the collection of holes enclosed by $\gamma$ uniquely determines the image of $D_{\gamma}$ in the abelianization of the mapping class group, $\operatorname{Ab} \operatorname{Map} \mathbf{D}_{n}$.

It will be helpful to decompose all Dehn twists as follows: given a Dehn twist $D_{\gamma}$ around a convex curve $\gamma$ that encloses $r$ holes, we apply the lantern relation repeatedly to write $D_{\gamma}$ as a composition of Dehn twists around (convex) curves enclosing all possible pairs of holes among the given $r$, together with some Dehn twists around single holes. Each pair of holes will contribute exactly one positive convex Dehn twist into this decomposition; each hole will have $r-2$ negative Dehn twists around it. Similarly, we can decompose an arbitrary Dehn twist in the same fashion; however, the Dehn twists enclosing pairs of holes will no longer have to be convex. We will refer to Dehn twists around a pair of holes as "pairwise" Dehn twists, and to Dehn twists around single holes as boundary twists. (The Dehn twist around the outer boundary component of the disk will not be referred to as a boundary twist.)

Now we can decompose an arbitrary element $\phi \in \operatorname{Map} \mathbf{D}_{n}$ : write it as a product of Dehn twists, and decompose each of them as above. (We will not be recording the order of Dehn twists in the monodromy.) Let $m_{i j}(\phi)$ be the multiplicity of the positive Dehn twist containing only the $i$-th and $j$-th holes in the decomposition of $\phi$; similarly, $m_{i}(\phi)$ be the multiplicity of the positive Dehn twist $\delta_{i}$ around the $i$-th hole. The collection of integers $m_{i j}, m_{i}$ is well-defined for $\phi \in \operatorname{Map} \mathbf{D}_{n}$ : because the multiplicities $m_{i j}, m_{i}$ are invariant under lantern relations, they do not depend on the factorization of $\phi$. Moreover, the collection $\left\{m_{i j}, m_{i}\right\}$ uniquely determines the image of $\phi$ in $\operatorname{Ab~Map} \mathbf{D}_{n}$.

Let $\Phi$ be the monodromy of the open book for $\left(L(p, 1), \xi_{k}\right)$ pictured in Figure 3 . Assume that $\Phi$ is factored into a product of some positive Dehn twists. Pick the $k$-th hole, which is enclosed by both curves $\alpha$ and $\beta$ in Figure 3, and consider the Dehn twists around it in the new factorization of $\Phi$. We consider only those Dehn twists that enclose at least two holes; suppose there are $l$ of them, and write $n_{1}, n_{2}, \ldots, n_{l}$ for the number of holes they enclose. 
Now, we compute the multiplicities of the pairwise and boundary twists for $\Phi$ that involve the $k$-th hole. The Dehn twists $D_{\alpha}, D_{\beta}$ contribute $n-1$ positive pairwise Dehn twists involving $k$-th hole $\left(m_{i k}=1\right.$ for every $\left.i \neq k\right)$ and $(k-2)+(n-k+1-2)=n-3$ negative boundary twists around it. On the other hand, the $l$ positive Dehn twists contribute $\left(n_{1}-1\right)+\left(n_{2}-1\right)+\cdots+\left(n_{l}-1\right)$ positive pairwise Dehn twists involving the $k$-th hole, and $\left(n_{1}-2\right)+\left(n_{2}-2\right)+\ldots\left(n_{l}-2\right)$ negative boundary Dehn twists. As the new factorization of $\Phi$ may have some positive boundary twists $D_{\delta_{k}}$ that we haven't yet taken into account,

$$
\begin{aligned}
n-1 & =\left(n_{1}-1\right)+\left(n_{2}-1\right)+\cdots+\left(n_{l}-1\right), \\
-((n-1)-2) & \geq-\left(n_{1}-2\right)-\left(n_{2}-2\right)-\cdots-\left(n_{l}-2\right) .
\end{aligned}
$$

Two cases are then possible:

(i) $l=2$, and the new positive factorization of $\Phi$ has no boundary twists around the $k$-th hole.

(ii) $l=1$, and there is exactly one positive boundary twist $D_{\delta_{k}}$ around the $k$-th hole.

To rule out the second case, notice that since $m_{i k}(\Phi)=1$ for every $i \neq k$, the unique positive Dehn twist in the decomposition of $\Phi$ would have to enclose all $n$ holes. But then we would have $m_{1 n}(\Phi)=1$, which contradicts the original definition of $\Phi$ from Figure 3.

The first case then tells us that the new positive decomposition of $\Phi$ has exactly two twists enclosing more than one hole each; there may also be some positive boundary twists. Examining the multiplicities $m_{i j}(\Phi), m_{i}(\Phi)$ again, we see that one Dehn twist must be around a curve that encloses the holes $1,2, \ldots k$, the other around a curve that encloses the holes $k, k+1, \ldots n$; denote the first curve by $\alpha^{\prime}$, the second by $\beta^{\prime}$. In addition, there must be one positive boundary twist $D_{\delta_{i}}$ around $i$-th hole for each $i \neq k$.

Lemma 2.3 The open book whose monodromy is the product

$$
D_{\alpha^{\prime}} D_{\delta_{1}} \cdots D_{\delta_{k-1}} D_{\delta_{k+1}} \cdots D_{\delta_{n}}
$$

represents $\left(S^{3}, \xi_{\text {std }}\right)$. The knot in $S^{3}$ induced by $\beta^{\prime}$ is the unknot; the framing on this unknot induced by the page framing of $\beta^{\prime}$ is $-p+1$ (compared to the Seifert framing).

Proof We can always find a self-diffeomorphism of the disk that maps $\alpha^{\prime}$ to $\alpha$, so we may assume that the curve $\alpha^{\prime}$ is standard. (Note, however, that we cannot 
simultaneously map $\alpha^{\prime}$ to $\alpha$ and $\beta^{\prime}$ to $\beta$ ). The open book with the monodromy $D_{\alpha^{\prime}} D_{\delta_{1}} \cdots D_{\delta_{k-1}} D_{\delta_{k+1}} \cdots D_{\delta_{n}}$ is obtained from the standard open book for $\left(S^{3}, \xi_{\text {std }}\right)$ with annular pages by $n-1$ stabilizations, so it represents the standard tight $S^{3}$ as well.

Next, we compute the page framing of the knot induced by $\beta^{\prime}$; or rather, we will compute the surgery framing, which is the page framing minus 1 . (We know that surgery on the corresponding knot yields $L(p, 1)$, so the surgery framing must be $\pm p$, but the sign needs to be determined.) Consider the Kirby diagram corresponding to the open book, Figure 4. We will destabilize the open book, starting with the holes

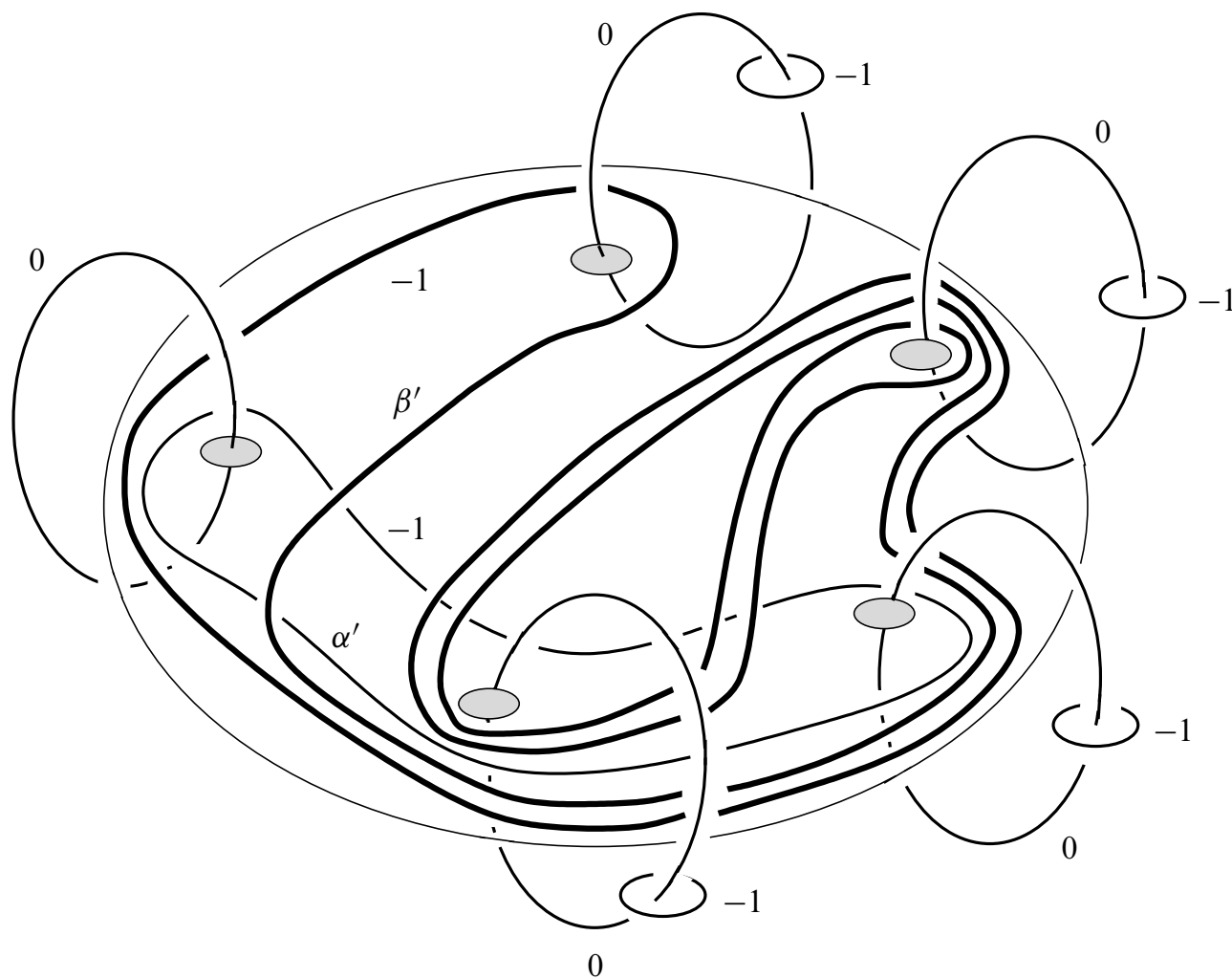

Figure 4: Surgery diagram for the open book

enclosed by $\beta^{\prime}$ and not by $\alpha^{\prime}$. The knot $\beta^{\prime}$ will no longer lie on the page; in the Kirby diagram, destabilizations amount to blowdowns shown in Figure 5.

After $n-k=p-1-k$ blowdowns, the framing decreases by $n-k$. The knot $\beta^{\prime}$ has the linking number 1 with the 0 -framed unknot corresponding to the $k$-th hole (the one enclosed by both $\alpha$ and $\beta$ ), and the linking number zero with every other component 


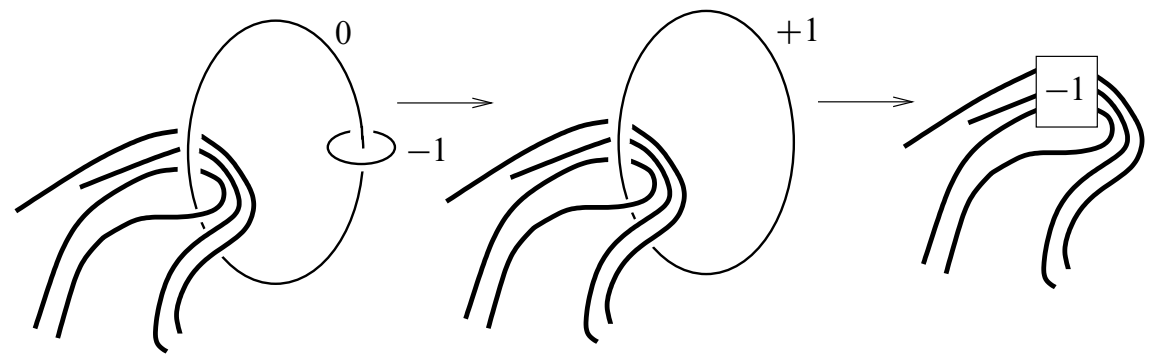

Figure 5: Blowing down to destabilize at holes enclosed by $\beta^{\prime}$
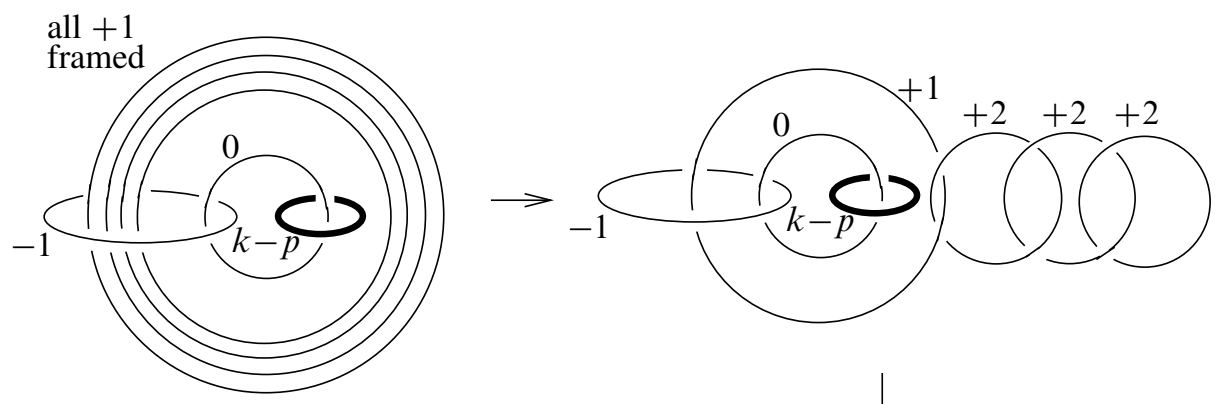

$$
\downarrow
$$

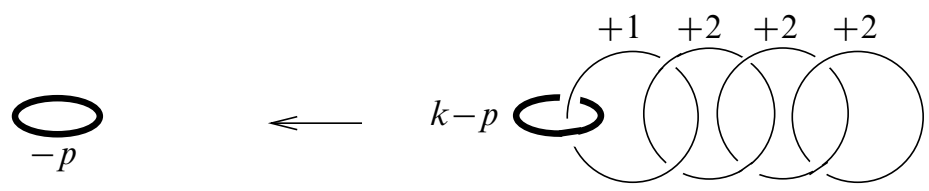

Figure 6: Computing the framing. The thick circle represents the knot induced by $\beta^{\prime}$, and is to be understood schematically: a priori it doesn't have to be an unknot, and its geometric linking with other components may be more complicated. However, the linking numbers are as shown: the knot $\beta^{\prime}$ has $l k=1$ with the 0 -framed component, and zero linking numbers with the rest. It follows that the framings will change as dictated by Kirby moves in the picture.

of the surgery link. (Note that the geometric linking may be quite complicated.) Next, we perform further Kirby moves as in Figure 6; knowing all linking numbers in the picture suffices for the framing calculation, even if the topological type of the knot $\beta^{\prime}$ is unknown. It follows that the surgery framing of the original knot induced in $S^{3}$ by the curve $\beta^{\prime}$ is $-p$. To see that $\beta^{\prime}$ must be the unknot, we invoke a theorem of Kronheimer-Mrowka-Ozsváth-Szabó [16] that states that the result of a $-p$-surgery on a knot in $S^{3}$ can be the lens space $L(p, 1)$ only if the knot is the unknot. 
Proof of Theorem 1.2 Lemma 2.3 implies that the Lefschetz fibration $X$ corresponding to the factorization of the monodromy as the product $D_{\alpha^{\prime}} D_{\delta_{1}} \cdots D_{\delta_{k-1}} D_{\delta_{k+1}}$ $\cdots D_{\delta_{n}} D_{\beta^{\prime}}$ is diffeomorphic to $D^{4}$ with a 2 -handle attached along a $-p$ framed unknot. (Note that the order of Dehn twists in this product is not important.) This is the standard filling of $L(p, 1)$, the disk bundle with Euler number $-p$. Moreover, $X$ has a Stein structure that arises from the Legendrian surgery on a Legendrian representative of the unknot $\beta^{\prime}$, with $t b=-p+1$. Since Legendrian unknots are classified by their Thurston-Bennequin and rotation numbers [4], we know that the only unknot that can produce $\left(L(p, 1), \xi_{k}\right)$ is the one shown in Figure 3, up to Legendrian isotopy. Because a compatible symplectic structure on a Lefschetz fibration is unique up to symplectic deformation [10], it follows that all Stein structures on $X$ are symplectic deformation equivalent; by Theorem 1.1, this means that the Stein filling of $(L(p, 1), \xi)$ is unique up to symplectic deformation, and any strong symplectic filling is unique up to symplectic deformation and blow-up.

Finally, recall that every weak filling of a rational homology sphere can be modified into a strong filling [23]; it follows that the weak symplectic filling of $(L(p, 1), \xi)$ is also unique, up to deformation and blow-up.

Remark 2.4 A very similar argument gives a new proof of McDuff's result [22] on uniqueness of filling for the standard (universally tight) contact structure on $L(p, 1)$ for $p \neq 4$. The page of the corresponding open book is a disk with $n=p-1$ holes; the monodromy is the product of positive Dehn twists around the holes (one twist for each hole) and the positive Dehn twist around the outer boundary component. Decomposing this monodromy, we get one positive Dehn twist around each pair of holes, and $n-3$ negative boundary twists for each hole. If there's a different positive factorization with $l$ nonboundary Dehn twists involving an arbitrary fixed hole and enclosing respectively $n_{1}, n_{2}, \ldots, n_{l}$ holes, we decompose them as before to see that Equations (1) and (2) must again hold. It follows that a positive factorization must either be the one we started with, or it must have two "nonboundary" Dehn twists involving each puncture, with no boundary twists. When $n \neq 3$, the second case is not possible, because otherwise some "pairwise" Dehn twists would not be present in the decomposition. It follows that there is a unique factorization of the monodromy, with positive boundary twists around each hole and one positive twist that encloses all the holes. (Unlike the case with two nonboundary twists considered above, no application of the deep result of [16] is needed here.) The classification of Legendrian unknots completes the proof as before; we see that the Stein filling is unique up to symplectic deformation. (This is weaker than Hind's result [12].)

When $n=3$ (ie $p=4$ ), an alternate positive factorization of the given monodromy indeed exists. It is given by the classical lantern relation, and corresponds to a nonstandard 

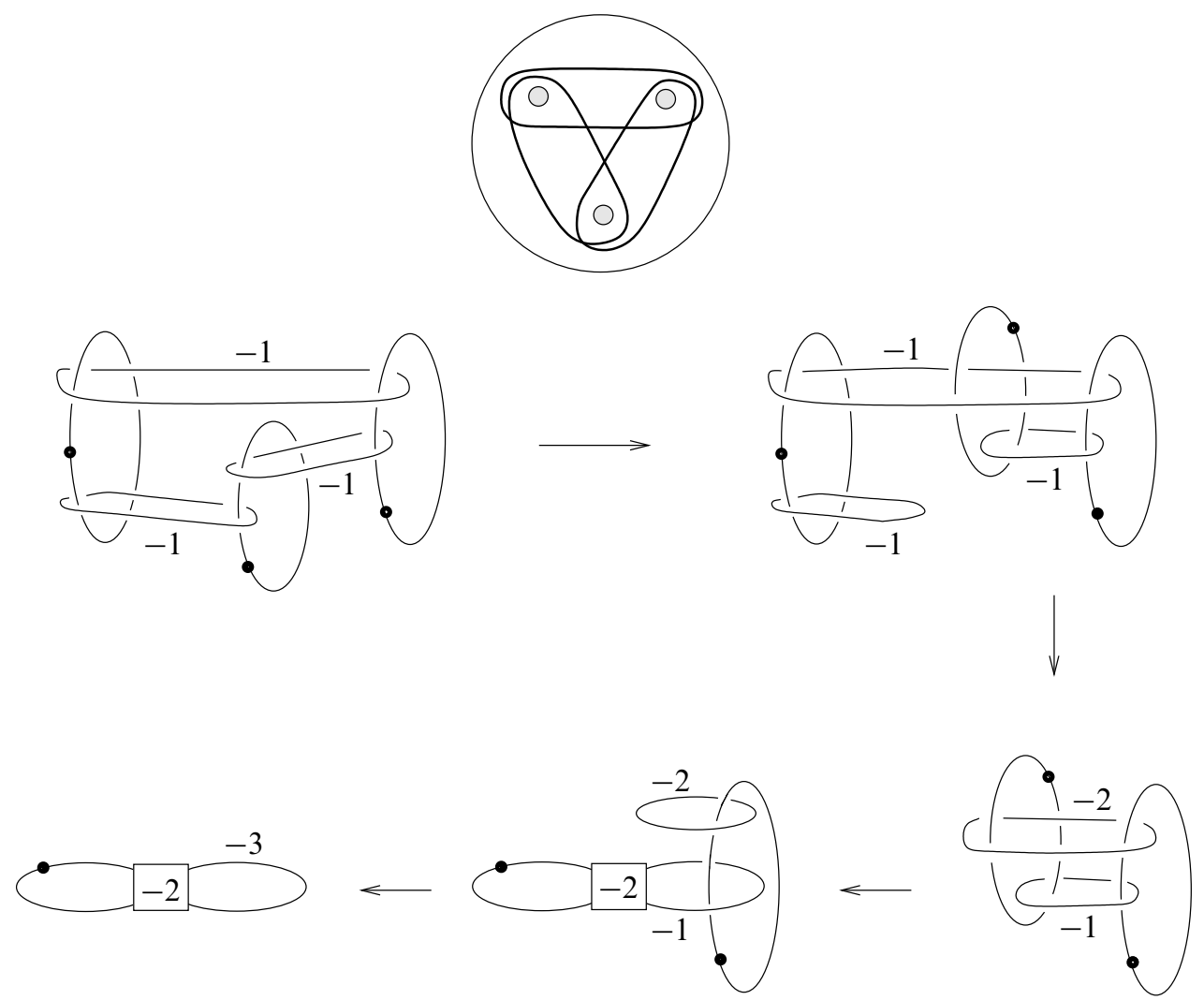

Figure 7: Using the lantern relation to construct a nonstandard Stein filling of $L(4,1)$

filling of $\left(L(4,1), \xi_{\text {std }}\right)$ which is a rational homology ball. (See Figure 7 for a Kirby calculus picture demonstrating that the lantern relation produces the nonstandard filling constructed in $[22 ; 18]$.) Note that we do not check that the nonstandard filling is unique: indeed, the images of all the Dehn twists in $A b \operatorname{Map} \mathbf{D}_{n}$ are uniquely determined, but we do not have an appropriate analog of Lemma 2.3 for this case.

Remark 2.5 The same strategy proves uniqueness of fillings for the universally tight contact structures on $L(p k+1, p)$, provided that $p, k \geq 1$, and either $k \leq p-2$, or $p=2$. See Figure 8 , where $p$ is the number of cusps in the surgery diagram (and the number of holes in the page of the open book); $k-1$ is the number of standard unknots (and the number of the multiple boundary twists). If $k>p-2>0$, a lantern-type relation (see Figure 11) can be used to construct a Stein filling different from the one given by the surgery diagram. These contact structures are covered by Lisca's 

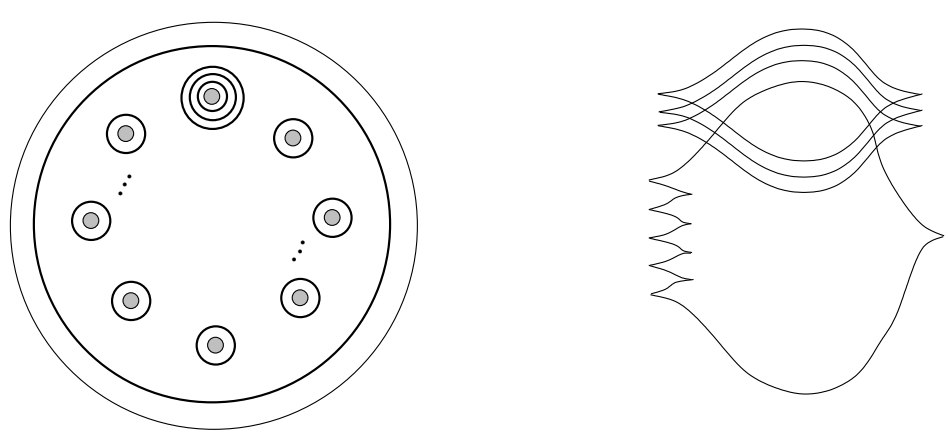

Figure 8: Universally tight contact structures on $L(p k+1, p)$

work [18], but our technique gives a slightly stronger result: uniqueness of filling up to symplectic deformation, not just diffeomorphism.

\section{Nonfillable contact structures on Seifert fibered spaces}

In this section we examine fillability of contact structures on the spaces $M\left(-1, r_{1}, r_{2}, r_{3}\right)$. All of these contact structures can be represented by planar open books; using Wendl's theorem, we can prove that a contact structure is nonfillable (in the strong symplectic sense) by showing that its monodromy admits no positive factorization in the mapping class group of a disk with holes. Since all of the spaces we consider are rational homology spheres, weak symplectic nonfillability follows as well.

Interestingly, our obstructions vanish for a family of open books, and the contact manifolds $M\left(-1, r_{1}, r_{2}, r_{3}\right)$ corresponding to this family turn out to be Stein fillable. This is not obvious from the given open books, as their monodromies are compositions of both positive and negative Dehn twists. To establish fillability, we rewrite these monodromies as products of positive Dehn twists by applying a generalized lantern relation that we develop in Lemma 3.5. (In fact, it suffices to prove fillability of tight contact structures $M(-1 ;(p-1) / p, 1 / 2,1 / p)$, since all the contact structures in our family arise via Legendrian surgeries on these.)

To obstruct the existence of a positive factorization, we work with abelianization of the mapping class group as before. There we can completely determine the cone of positive mapping classes. The complete picture can be difficult to use in general, and so we extract some simpler invariants which are easier to use. The methods used here generalize easily and can in practice be quite simple to apply to a given open book. We believe that the techniques here will be useful to those looking to determine the tightness or fillability of a contact structure given as a planar open book. 
The following lemma will be used repeatedly to control the number of Dehn twists in possible positive factorizations.

Lemma 3.1 Let $\phi \in \operatorname{Map}_{n}$ be given as a product of (positive or negative) Dehn twists. Suppose a hole $q$ is enclosed by $b$ boundary and $k$ nonboundary twists. Here $b$ and $k$ are the algebraic or signed counts of Dehn twists in a presentation of $\phi$. Then in any positive factorization of $\phi$ there are no more than $k+b$ nonboundary Dehn twists enclosing $q$.

Proof We generalize Equations (1)-(2). Suppose $\phi$ is given as a factorization into Dehn twists involving $k_{+}$positive and $k_{-}$negative Dehn twists around $q$, so that $k=k_{+}-k_{-}$. Further suppose the $k_{+}$positive Dehn twists enclose $m_{1}, m_{2}, \ldots, m_{k_{+}}$ holes, while the $k_{-}$negative Dehn twists enclose respectively $M_{1}, M_{2}, \ldots, M_{k_{-}}$holes each. Assume that $\phi$ has a positive factorization where the hole $q$ is enclosed by $l$ nonboundary positive Dehn twists around resp. $n_{1}, n_{2}, \ldots, n_{l}$ holes. Computing the multiplicity $m_{q}$ of the boundary twist around $q$ in the decomposition of $\phi$, as well as the pairwise multiplicities $m_{q q^{\prime}}$ of all pairs that involve $q$, we have

$$
\begin{aligned}
\left(m_{1}-1\right)+\cdots+\left(m_{k_{+}}-1\right)-\left(M_{1}-1\right)-\cdots-\left(M_{k_{-}}-1\right) & \\
& =\left(n_{1}-1\right)+\cdots+\left(n_{l}-1\right), \\
\left(M_{1}-2\right)+\cdots+\left(M_{k_{-}}-2\right)+b-\left(m_{1}-2\right)-\cdots-\left(m_{k_{+}}-2\right) & \\
& \geq-\left(n_{1}-2\right)-\cdots-\left(n_{l}-2\right) .
\end{aligned}
$$

It follows that $k+b=k_{+}-k_{-}+b \geq l$.

Example 3.2 To avoid the more tedious analysis of cases that we'll need later on, we begin with an example taken from [8, Figure 7], which is a tight contact structure $\Xi$ on the Seifert fibered space $M=M(-1 ; 1 / 2,1 / 2,1 / p)$. The contact structure $\Xi$ is given by the surgery diagram on the left of Figure 9; we can convert it into an open book on the right. (The order of Dehn twists is unimportant for this particular product.) It is shown in [8] that for $p>2(M, \Xi)$ is not Stein fillable, and that it is not symplectically fillable for $p \not \equiv 2 \bmod 8$. Since $(M, \Xi)$ can be represented by a planar open book, Stein nonfillability together with Wendl's work immediately implies that $(M, \Xi)$ is not symplectically fillable for all $p>2$. We now obtain an alternative quick proof of nonfillability, using our technique.

The monodromy $\Phi$ of the open book representing $(M, \Xi)$ is the product of several positive and one negative Dehn twist. There are $p-1 \geq 2$ holes outside of the negative Dehn twist; denote them by $s_{1}, \ldots, s_{p-1}$. Suppose that $\Phi$ is factored into a product of positive Dehn twists around some curves; let $\left\{D_{\alpha}\right\}$ be the set of nonboundary twists in 

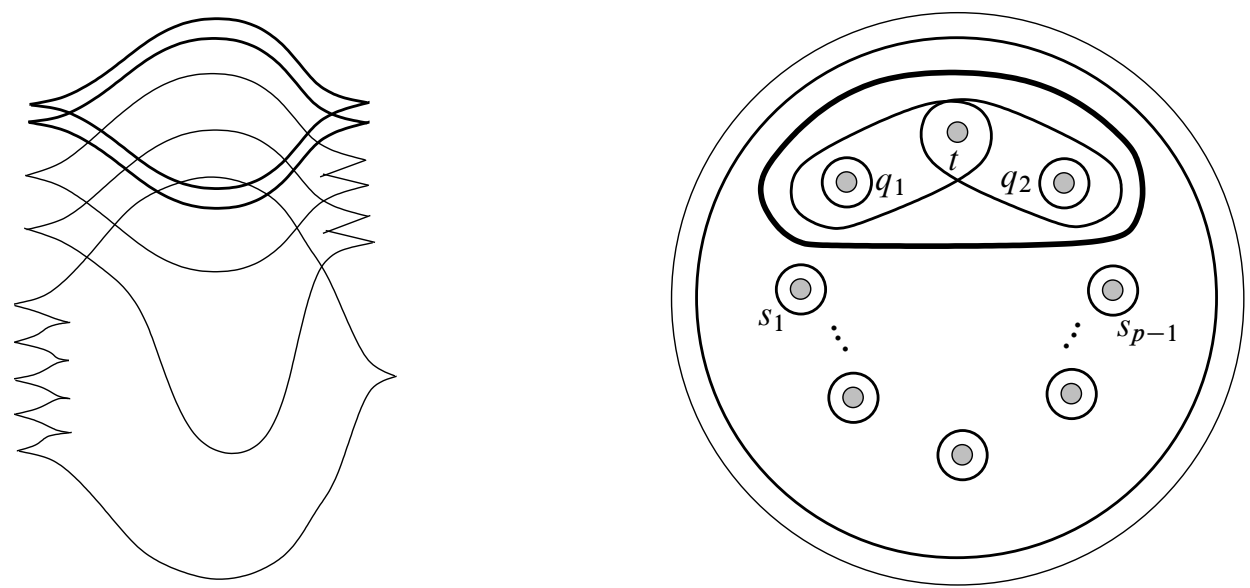

Figure 9: The nonfillable tight contact structure on $M(-1 ; 1 / 2,1 / 2,1 / p)$. The surgery diagram has two +1 contact surgeries (on the thicker Legendrian unknots); the rest are Legendrian surgeries. Accordingly, the monodromy is the product of one negative Dehn twist (around the thicker curve) and many positive ones.

this factorization. Lemma 3.1 implies that every hole in the picture can be enclosed by no more than two of $D_{\alpha}$ 's. Moreover, the pair of holes $q_{1}$ and $q_{2}$ is enclosed by one positive and one negative Dehn twist, so the multiplicity $m_{q_{1} q_{2}}$ is zero. This means that none of $D_{\alpha}$ 's can enclose both $q_{1}$ and $q_{2}$.

Now, consider the hole $s_{1}$. Since the pairs $\left\{q_{1}, s_{1}\right\},\left\{q_{2}, s_{1}\right\},\left\{t, s_{1}\right\}$ must all be enclosed with multiplicity $1, q_{1}$ and $q_{2}$ cannot be enclosed together, and $s_{1}$ is enclosed by no more than two nonboundary twists, we must have a twist $D_{\alpha_{1}}$ that encloses $t, q_{1}$, and $s_{1}$ (but not $q_{2}$ ), and another twist $D_{\alpha_{2}}$ that encloses $q_{2}$ and $s_{1}$ (but not $q_{1}$ and $t$ ). (The roles of $q_{1}$ and $q_{2}$ may be reversed). See Figure 10. Because the pair $\left\{q_{2}, t\right\}$ has multiplicity 1 , there must also be the twist $D_{\alpha_{3}}$ that encloses $t$ and $q_{2}$ but not $q_{1}$ and $s_{1}$. Next, consider the hole $s_{2}$. Since $m_{s_{1} s_{2}}=1$, and $D_{\alpha_{1}}, D_{\alpha_{2}}$ are the only nonboundary twists around $s_{1}$, the hole $s_{2}$ is enclosed by exactly one of $D_{\alpha_{1}}$ and $D_{\alpha_{2}}$. If $s_{2}$ were in $D_{\alpha_{1}}$, we would have $m_{t s_{2}}=2$ (if $s_{2}$ is in $D_{\alpha_{3}}$ ), or $m_{q_{2} s_{2}}=0$ (if it isn't). Similarly, if $s_{2}$ were in $D_{\alpha_{2}}$, we have $m_{t s_{2}}=2$ or $m_{q_{1} s_{2}}=0$. The contradiction shows that a positive factorization of $\Phi$ can't exist.

Remark 3.3 When $p=2$, the corresponding contact structure $\Xi$ is Stein fillable. This can be seen from the open book: indeed, if there are no additional holes $s_{2}, \ldots, s_{k}$, by the lantern relation Figure 10 provides a positive factorization of the monodromy as the product $D_{\delta} D_{\alpha_{3}} D_{\alpha_{2}} D_{\alpha_{1}}$, where $D_{\delta}$ is the boundary twist around the hole $q_{1}$. 


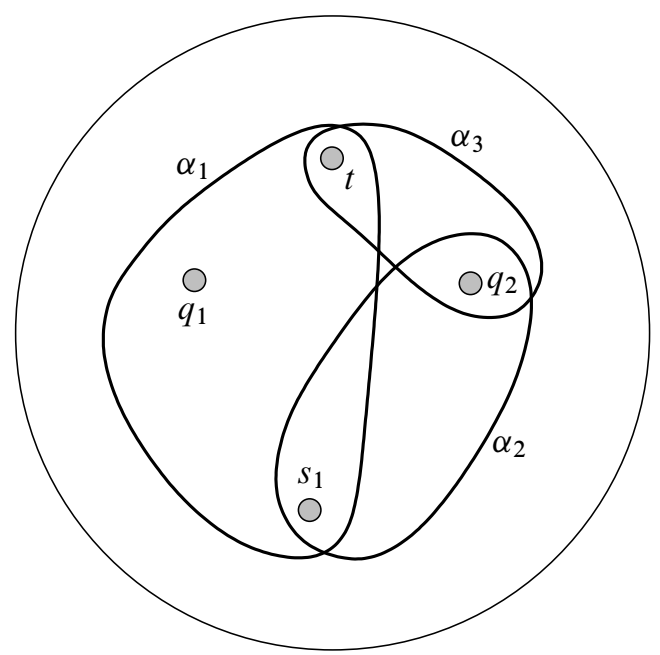

Figure 10: Trying to construct a positive factorization for the monodromy of the open book from Figure 9

The above argument readily generalizes to some more complicated open books representing contact structures on other spaces $M\left(-1 ; r_{1}, r_{2}, r_{3}\right)$.

Recall that tight contact structures on the Seifert fibered space $M\left(-1 ; r_{1}, r_{2}, r_{3}\right)$, where $r_{1}, r_{2} \geq 1 / 2$, were classified in [8] and can be described as follows. The space $M(-1 ; 1 / 2,1 / 2,1 / p)$ carries exactly three tight contact structures for each $p>2$; two of them are Stein fillable, the third is the nonfillable contact structure $\Xi$ we studied in Example 3.2. It was shown in [8] that for $r_{1}, r_{2} \geq 1 / 2$, all tight contact structures on $M\left(-1 ; r_{1}, r_{2}, r_{3}\right)$ can be obtained via Legendrian surgeries on these three contact structures. The Legendrian surgeries arise from the continued fraction expansions of $r_{1}, r_{2}, r_{3}$; thus, the surgeries are performed on chains of Legendrian unknots, with coefficients $a_{i}, b_{i}, c_{i}$. The Legendrian unknots must be stabilized accordingly, to match $\mathrm{tb}-1$ and the surgery coefficients. In general, many choices for stabilizations may be possible. Fillability needs to be investigated only for those contact structures obtained from $\Xi$, as all others will automatically be fillable.

We first prove the "fillable" part of Theorem 1.4. Indeed, suppose $c_{1}=p \leq \max \left(k_{1}, k_{2}\right)$. We can assume $k_{1} \geq k_{2}$; this assumption implies $\left[a_{1}, a_{2}, \ldots, a_{p-1}\right]=[2,2, \ldots, 2]=$ $(p-1) / p$. In that case, all the tight contact structures on $M\left(-1 ; r_{1}, r_{2}, r_{3}\right)$ are obtained from $M(-1 ;(p-1) / p, 1 / 2,1 / p)$ by Legendrian surgeries. Thus, it suffices to prove the following:

Proposition 3.4 All tight contact structures on $M(-1 ;(p-1) / p, 1 / 2,1 / p)$ are Stein fillable. 

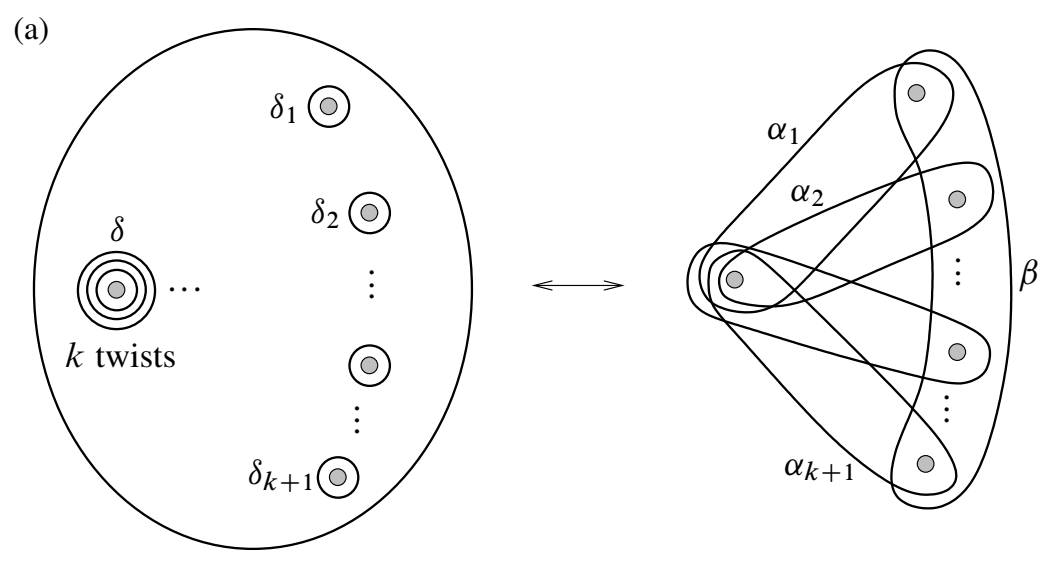

(b)

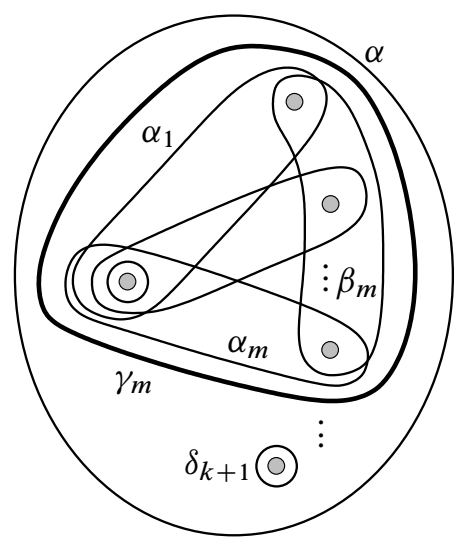

Figure 11: (a) Generalized lantern relation for a disk with $k+2$ holes. (b) Proving it inductively: After $m-1$ applications of the classical lantern relation, the monodromy from the top right picture can be written as $k-m+1$ positive Dehn twists around $\delta$, a negative twist around $\gamma_{m}$, and positive twists around $\alpha_{1}, \alpha_{2}, \ldots, \alpha_{m}, \beta_{m}$, where the curve $\beta_{m}$ encloses $m$ holes.

Proof We only need to check fillability of the (unique) contact structure on the manifold $M(-1 ;(p-1) / p, 1 / 2,1 / p)$ obtained by Legendrian surgery on $\Xi$. Its contact surgery diagram is shown on the left of Figure 12; to build the open book, we translate the chains of small unknots in the surgery diagram into sequences of push-offs of the stabilized unknot they are linked to. This is possible because the standard Legendrian meridian and the push-off of a Legendrian knot are Legendrian isotopic in the manifold obtained by Legendrian surgery on this knot [2]. The resulting open book is on the right of Figure 12. To prove the proposition, we rewrite the monodromy as a product of positive Dehn twists using the lantern-type relation of Lemma 3.5 below. 

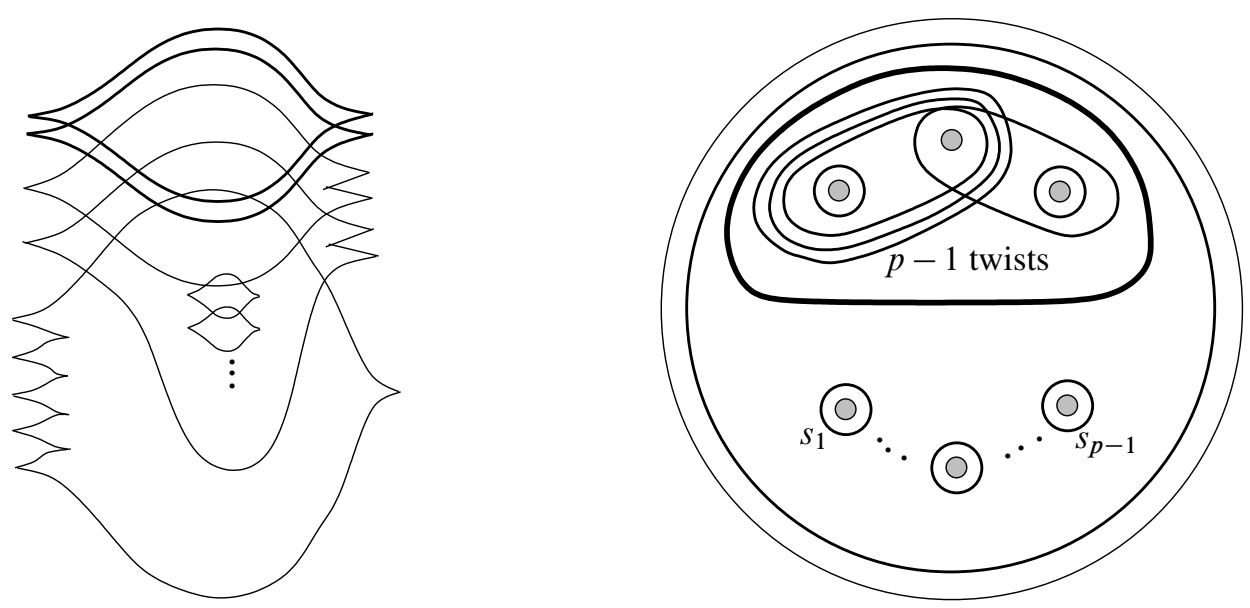

Figure 12: A fillable contact structure on $M(-1 ;(p-1) / p, 1 / 2,1 / p)$

Lemma 3.5 In the mapping class group of the disk with $k+2$ holes, the relation

$$
\left(D_{\delta}\right)^{k} D_{\delta_{1}} D_{\delta_{2}} \cdots D_{\delta_{k+1}} D_{\alpha}=D_{\beta} D_{\alpha_{k+1}} \cdots D_{\alpha_{2}} D_{\alpha_{1}}
$$

holds for positive Dehn twists around curves shown in Figure 11(a). Our convention for products means that in the right-hand side, $D_{\beta}$ is performed first.

Proof The classical lantern relation, together with an inductive argument, shows that the relation

$$
\left(D_{\delta}\right)^{k} D_{\alpha} D_{\delta_{1}} D_{\delta_{2}} \cdots D_{\delta_{k}+1}=\left(D_{\delta}\right)^{k-m+1} D_{\alpha}\left(D_{\gamma_{m}}\right)^{-1} D_{\beta_{m}} D_{\alpha_{m}} \cdots D_{\alpha_{2}} D_{\alpha_{1}}
$$

holds for each $m, k+1 \geq m \geq 2$. The right-hand side of this identity is illustrated on Figure 11(b); note that $\gamma_{k+1}=\alpha$.

It remains to prove the nonfillability part of Theorem 1.4. To this end, we construct the candidate nonfillable structures as follows. As before, we consider contact structures obtained by Legendrian surgery on $(M(-1 ; 1 / 2,1 / 2,1 / p), \Xi)$ (for various values $p>2)$. The coefficients $a_{1}, \ldots, a_{k_{1}}, b_{1}, \ldots, b_{k_{2}}$ are equal to 2 and thus correspond to standard unknots with no stabilizations. If $k_{1}<n_{1}$ or $k_{2}<n_{2}$, there are some coefficients $a_{k_{1}+1}, b_{k_{2}+1}, \ldots$ that are greater than 2 . This means that the corresponding Legendrian unknots must be stabilized; we assume that all stabilizations are chosen on the left. Similarly, we stabilize on the left all the unknots corresponding to the continued fraction expansion $r_{3}=\left[c_{1}, c_{2}, \ldots c_{n_{3}}\right]$; note that $c_{1}=p$ if our contact structure is obtained from $(M(-1 ; 1 / 2,1 / 2,1 / p), \Xi)$. The resulting contact structure $\xi$ on $M\left(-1 ; r_{1}, r_{2}, r_{3}\right)$ is given by the contact surgery diagram and the open book shown 

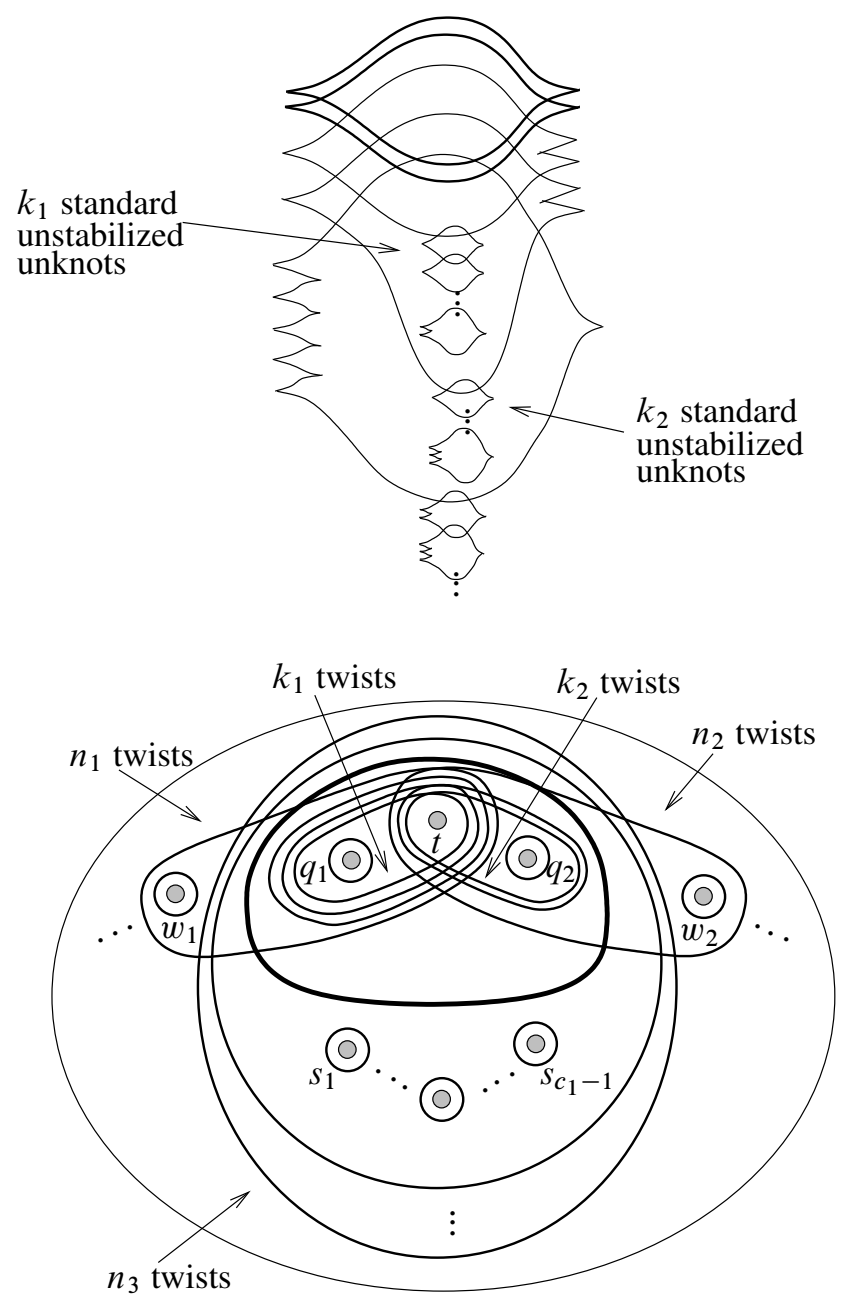

Figure 13: Nonfillable tight contact structures on $M\left(-1 ; r_{1}, r_{2}, r_{3}\right)$, with $1>r_{1}, r_{2} \geq 1 / 2$

in Figure 13. (As in Proposition 3.4, we use the identification of the meridian and the push-off of a Legendrian knot in a surgered manifold [2] to translate the chains of small unknots in the surgery diagram into sequences of iterated and possibly stabilized push-offs.)

Recall that $\xi$ is tight by [8] (indeed, the Heegaard Floer contact invariant $c(\Xi)$ is nonzero, so $c(\xi) \neq 0$ as well).

Proposition 3.6 The contact structure $\xi$ on $M\left(-1 ; r_{1}, r_{2}, r_{3}\right)$ described above and shown in Figure 13 is nonfillable. 
Proof We first point out several features of the open book representing $\xi$. The monodromy is the product of Dehn twists itemized below: there is a unique negative Dehn twist (explicitly mentioned in the list), and all other Dehn twists are positive.

- There is a collection of $n_{1}$ Dehn twists enclosing the holes $q_{1}$ and $t ; k_{1}$ of them enclose exactly these two holes, the extra $n_{1}-k_{1}$, if present, all enclose a hole $w_{1}$, but not $q_{2}$ or $s_{1}, s_{2}, \ldots, s_{c_{1}-1}$.

- There is a collection of $n_{2}$ Dehn twists enclosing the holes $q_{2}$ and $t ; k_{2}$ of them enclose exactly these two holes, an extra $n_{2}-k_{2}$, if present, all enclose a hole $w_{2}$, but not $q_{1}$ or $s_{1}, s_{2}, \ldots, s_{c_{1}-1}$.

- There is one negative Dehn twist enclosing three holes $q_{1}, q_{2}$ and $t$.

- There are $n_{3}$ Dehn twists enclosing $c_{1}+2$ holes $q_{1}, q_{2}, t$, and $s_{1}, s_{2}, \ldots, s_{c_{1}-1}$, and perhaps some additional holes (but not $w_{1}$ or $w_{2}$ ).

Our task is to show that this monodromy admits no positive factorization. We will do so by analyzing the Dehn twists that can enclose the holes $s_{i}$. First, notice that since the multiplicity of any pair $\left\{w_{1}, s_{i}\right\},\left\{w_{2}, s_{i}\right\}$ is zero, any Dehn twist in a positive factorization that encloses of the $s_{i}$ 's cannot enclose $w_{1}$ or $w_{2}$. We will refer to the nonboundary Dehn twists that contain neither $w_{1}$ nor $w_{2}$ as inner Dehn twists; the (nonboundary) Dehn twists containing $w_{1}$ or $w_{2}$ will be called outer twists, and will not play much role in our argument.

First, we will calculate multiplicities of pairs of holes and use Lemma 3.1 to establish the following properties of any possible positive factorization.

(a) Exactly $n_{3}-1$ inner Dehn twists contain both $q_{1}$ and $q_{2}$; all of these contain $t$.

(b) Exactly $k_{1}$ inner Dehn twists contain $q_{1}$ and $t$, but not $q_{2}$ (call these $t q_{1}$-twists).

(c) Exactly $k_{2}$ inner Dehn twists contain $q_{2}$ and $t$ but not $q_{1}$ (call these $t q_{2}$-twists).

(d) There is at most one inner Dehn twist $D_{\alpha_{1}}$ that contains $q_{1}$ but not $q_{2}$ or $t$.

(e) There is at most one inner Dehn twist $D_{\alpha_{2}}$ that contains $q_{2}$ but not $q_{1}$ or $t$.

(f) Each of the holes $s_{1}, s_{2}, \ldots, s_{c_{1}-1}$ is contained in exactly $n_{3}+1$ nonboundary Dehn twists, exactly $n_{3}$ of which enclose $t$, exactly $n_{3}$ enclose $q_{1}$ and exactly $n_{3}$ enclose $q_{2}$.

(g) Any two holes $s_{i}, s_{j}$ are enclosed together by exactly $n_{3}$ Dehn twists.

To prove (a), observe that the multiplicity of the pair $\left\{q_{1}, q_{2}\right\}$ is $n_{3}-1$, which means there must be $n_{3}-1$ Dehn twists enclosing both holes. Each of them is an inner twist, since $m_{q_{2}, w_{1}}=m_{q_{1}, w_{2}}=0$. To see that all of these contain $t$, notice that by 
Lemma 3.1 there are at most $n_{1}+n_{2}+n_{3}-1$ Dehn twists containing $t ; n_{1}-k_{1}$ resp. $n_{2}-k_{2}$ of these must enclose $w_{1}$ resp. $w_{2}$, so there are at most $k_{1}+k_{2}+n_{3}-1$ inner Dehn twists containing $t$. Of these, at least $k_{1}+n_{3}-1$ contain $q_{1}$ (because $m_{q_{1} t}=n_{1}+n_{3}-1$, and $q_{1}$ can be in no more than $n_{1}-k_{1}$ outer Dehn twists containing $t$ and $w_{1}$, and in none containing $w_{2}$ ). Similarly, at least $k_{2}$ contain $q_{2}$. Since all three holes $t, q_{1}$, and $q_{2}$ can be enclosed together by no more than $n_{3}-1$ inner twists, the statements (a), (b) and (c) follow.

To prove (d), observe that by Lemma 3.1 there are at most $n_{1}+n_{3}$ nonboundary Dehn twists enclosing $q_{1}$. Of these, $n_{1}-k_{1}$ are outer twists (containing $w_{1}$ ). This leaves at most $k_{1}+n_{3}$ inner twists enclosing $q_{1}$. By (a) and (b), at most one of these inner twists can contain neither $t$ nor $q_{2}$. The proof of (e) is similar.

Finally, (f) follows from the fact that $m_{q_{1} s_{i}}=m_{q_{2} s_{i}}=m_{s_{i} t}=n_{3}$, together with (a) and Lemma $3.1 ;(\mathrm{g})$ is merely a multiplicity count.

We now show that properties (a)-(f) cannot hold if $c_{1}-1>\max \left(k_{1}, k_{2}\right)$. Indeed, (f), (a), (d) and (e) imply that each of the holes $s_{i}$ is enclosed by $n_{3}-1$ twists containing the three holes $t, q_{1}, q_{2}$ (these are the twists described in (a)). For the remaining two twists enclosing $s_{i}$, there are two possibilities: (i) a $t q_{1}$-twist and $D_{\alpha_{2}}$, or (ii) a $t q_{2}$ twist and $D_{\alpha_{1}}$. Moreover, no two of the holes $s_{i}$ can be contained in the same $t q_{1}$-twist: they are also both contained in the $D_{\alpha_{2}}$ twist and in all the twists of (a), which contradicts (g). Similarly, no two $s_{i}$ 's can be contained in the same $t q_{2}$-twist. Because $c_{1}-1>\max \left(k_{1}, k_{2}\right)$, we conclude that there must be a nonempty subset of $\left\{s_{1}, s_{2}, \ldots, s_{c_{1}-1}\right\}$ for which (i) holds true, and a nonempty subset for which (ii) holds true. Pick a hole from the first subset and another from the second; since we've listed all the $n_{3}+1$ twists enclosing each of these holes, we see that this pair of holes is enclosed together only by the $n_{3}-1$ twists containing the three holes $t, q_{1}, q_{2}$. This is a contradiction with $(\mathrm{g})$.

Remark 3.7 In fact, we have established nonfillability for a much wider class of contact structures on Seifert spaces $M\left(-1 ; r_{1}, r_{2}, r_{3}\right)$ for which the condition $c_{1}-1>$ $\max \left(k_{1}, k_{2}\right)$ is satisfied. Indeed, we only used the hypothesis that the unknots corresponding to $a_{k_{1}+1}$ and $b_{k_{2}+1}$ have at least one stabilization on the left, and that the unknot corresponding to $c_{1}$ has more than $\max \left(k_{1}, k_{2}\right)$ stabilizations on the left. All the other stabilizations may be chosen arbitrarily.

\section{Concluding remarks}

In the previous section, we worked with the spaces $M\left(-1, r_{1}, r_{2}, r_{3}\right)$ such that $r_{1}, r_{2} \geq$ $1 / 2$ because this condition was essential for the classification results and for the proofs 
of tightness of [8]. In fact, with slightly more tedious case-by-case analysis, one can extend our nonfillability results to certain spaces $M\left(-1 ; r_{1}, r_{2}, r_{3}\right)$, with arbitrary $r_{1}, r_{2} \in(0,1)$. We can also consider Seifert fibered spaces with more than 3 singular fibers; it is not hard to give sufficient conditions for an open book similar to the one in Figure 13 to represent a nonfillable contact structure. One can also hope to understand fillability for a wider class of open books (not necessarily planar) by using the fiber sum construction of [26]. In many of these situations, contact structures can be shown to be nonfillable by easy combinatorial considerations in the abelianization of the mapping class groups of planar surfaces.

However, nonfillability does not seem to be an interesting property unless the contact structure is known to be tight. In most cases, a proof of tightness requires an application of Heegaard Floer homology. For the spaces $M\left(-1, r_{1}, r_{2}, \ldots, r_{k}\right)$, nonvanishing of the Heegaard Floer contact invariant (and thus tightness) can be checked via the criterion of [19, Theorem 1.2], although this often requires lengthy calculations related to Heegaard Floer homology (see [20]).

It would be interesting to find hypotheses on the monodromy of a planar open book that ensure tightness of the corresponding contact structure. One related condition is the right-veering property of an open book [14]; indeed, a contact structure is tight if and only if every compatible open book is right-veering. In general, one needs to consider all compatible open books; indeed, by stabilizing an arbitrary open book, one can always obtain a right-veering open book representing the given contact structure. This is shown in [14, Proposition 6.1]; in fact, the proof of that proposition shows that stabilizations can be done without increasing the genus of the open book (but increasing the number of its boundary components). Thus, every contact structure supported by a planar open book can be supported by a right-veering planar open book. In fact, one can perform additional stabilizations to increase the pairwise multiplicities of the monodromy; it is not hard to show that every contact structure supported by a planar open book is supported by a planar open book with right-veering monodromy and positive pairwise multiplicities. By contrast, recall that a contact structure supported by an open book of genus one with one boundary component is tight if this particular open book is right-veering [15]; one can hope that since planar open books are another very special case, some sufficient conditions for a given monodromy to ensure tightness can be found.

In another direction, it would be interesting to generalize the classification results of Section 1. Indeed, it is possible to analyze positive factorizations in the abelianization of the mapping class group for a wide class of contact structures. However, we have no analogs of Lemma 2.3 (in fact no analogs of the theorem of [16]), and this poses a major obstacle for further classification results. 


\section{References}

[1] S Akbulut, B Ozbagci, Lefschetz fibrations on compact Stein surfaces, Geom. Topol. 5 (2001) 319-334 MR1825664

[2] F Ding, H Geiges, Handle moves in contact surgery diagrams, J. Topol. 2 (2009) 105-122 MR2499439

[3] Y Eliashberg, Filling by holomorphic discs and its applications, from: "Geometry of low-dimensional manifolds, 2 (Durham, 1989)", (S K Donaldson, C B Thomas, editors), London Math. Soc. Lecture Note Ser. 151, Cambridge Univ. Press (1990) 45-67 MR1171908

[4] Y Eliashberg, M Fraser, Classification of topologically trivial Legendrian knots, from: "Geometry, topology, and dynamics (Montreal, PQ, 1995)", (F Lalonde, editor), CRM Proc. Lecture Notes 15, Amer. Math. Soc. (1998) 17-51 MR1619122

[5] J B Etnyre, Planar open book decompositions and contact structures, Int. Math. Res. Not. (2004) 4255-4267 MR2126827

[6] J B Etnyre, Lectures on open book decompositions and contact structures, from: "Floer homology, gauge theory, and low-dimensional topology", (D A Ellwood, P Ozváth, A I Stipsicz, Z Szabó, editors), Clay Math. Proc. 5, Amer. Math. Soc. (2006) 103-141 MR2249250

[7] P Ghiggini, P Lisca, A I Stipsicz, Classification of tight contact structures on small Seifert 3-manifolds with $e_{0} \geq 0$, Proc. Amer. Math. Soc. 134 (2006) 909-916 MR2180909

[8] P Ghiggini, P Lisca, A I Stipsicz, Tight contact structures on some small Seifert fibered 3-manifolds, Amer. J. Math. 129 (2007) 1403-1447 MR2354324

[9] E Giroux, Géométrie de contact: de la dimension trois vers les dimensions supérieures, from: "Proceedings of the International Congress of Mathematicians, Vol. II (Beijing, 2002)", Higher Ed. Press, Beijing (2002) 405-414 MR1957051

[10] R E Gompf, Toward a topological characterization of symplectic manifolds, J. Symplectic Geom. 2 (2004) 177-206 MR2108373

[11] R E Gompf, A I Stipsicz, 4-manifolds and Kirby calculus, Graduate Studies in Math. 20, Amer. Math. Soc. (1999) MR1707327

[12] R Hind, Stein fillings of lens spaces, Commun. Contemp. Math. 5 (2003) 967-982 MR2030565

[13] K Honda, On the classification of tight contact structures. I, Geom. Topol. 4 (2000) 309-368 MR1786111

[14] K Honda, W H Kazez, G Matić, Right-veering diffeomorphisms of compact surfaces with boundary, Invent. Math. 169 (2007) 427-449 MR2318562 
[15] K Honda, W H Kazez, G Matić, On the contact class in Heegaard Floer homology, J. Differential Geom. 83 (2009) 289-311 MR2577470

[16] P Kronheimer, T Mrowka, P Ozsváth, Z Szabó, Monopoles and lens space surgeries, Ann. of Math. (2) 165 (2007) 457-546 MR2299739

[17] P Lisca, On symplectic fillings of 3-manifolds, from: "Proceedings of 6th Gökova Geometry-Topology Conference”, Turkish J. Math. 23 (1999) 151-159 MR1701644

[18] P Lisca, On symplectic fillings of lens spaces, Trans. Amer. Math. Soc. 360 (2008) 765-799 MR2346471

[19] P Lisca, A I Stipsicz, Ozsváth-Szabó invariants and tight contact 3-manifolds. III, J. Symplectic Geom. 5 (2007) 357-384 MR2413308

[20] P Lisca, A I Stipsicz, On the existence of tight contact structures on Seifert fibered 3-manifolds, Duke Math. J. 148 (2009) 175-209 MR2524494

[21] D Margalit, J McCammond, Geometric presentations for the pure braid group, J. Knot Theory Ramifications 18 (2009) 1-20 MR2490001

[22] D McDuff, The structure of rational and ruled symplectic 4-manifolds, J. Amer. Math. Soc. 3 (1990) 679-712 MR1049697

[23] H Ohta, K Ono, Simple singularities and topology of symplectically filling 4-manifold, Comment. Math. Helv. 74 (1999) 575-590 MR1730658

[24] P Ozsváth, A Stipsicz, Z Szabó, Planar open books and Floer homology, Int. Math. Res. Not. (2005) 3385-3401 MR2200085

[25] O Plamenevskaya, Contact structures with distinct Heegaard Floer invariants, Math. Res. Lett. 11 (2004) 547-561 MR2092907

[26] C Wendl, Contact fiber sums, monodromy maps, and symplectic fillings, in preparation

[27] C Wendl, Strongly fillable contact manifolds and J-holomorphic foliations, Duke Math. J. 151 (2010) 337-384 MR2605865

[28] H Wu, Tight contact small seifert spaces with $e_{0} \neq 0,-1,-2$ arXiv: math.GT/0402167

Department of Mathematics, Stony Brook University

Stony Brook NY 11794-3651, USA

American Institute of Mathematics

Palo Alto CA 94306, USA

olga@math.sunysb.edu, jvanhorn@aimath.org

Proposed: Yasha Eliashberg

Seconded: Peter Ozsvath, Leonid Polterovich

Received: 17 December 2009

Accepted: 13 August 2010 